\title{
Rational Design of the Nonlinear Optical Response in a Tin lodate Fluoride $\mathrm{Sn}\left(\mathrm{IO}_{3}\right)_{2} \mathrm{~F}_{2}$
}

Min Luo ${ }^{\dagger}$, Fei Liang ${ }^{\S}, \mathrm{Xia} \mathrm{Hao}^{\dagger, \star}$, Donghong Lin ${ }^{\dagger,}$, Bingxuan $\mathrm{Li}^{\dagger}$, Zheshuai Lin ${ }^{\S}$, and Ning $\mathrm{Ye}^{\dagger, \sigma *}$

†Key Laboratory of Optoelectronic Materials Chemistry and Physics, Collaborative Innovation Center for Optoelectronic Semiconductors and Efficient Devices, Fujian Institute of Research on the Structure of Matter, Chinese Academy of Sciences

${ }^{6}$ Fujian Science \& Technology Innovation Laboratory for Optoelectronic Information of China, Fuzhou, Fujian 350002, China

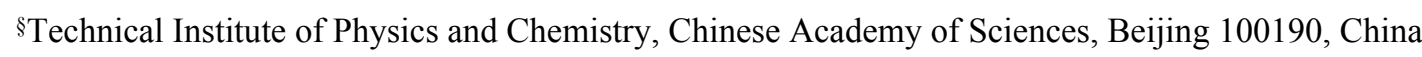
University of Chinese Academy of Sciences, Beijing 100190, China

Email: nye@fjirsm.ac.cn; 
Table of contents

\begin{tabular}{|c|c|c|}
\hline Sections & Titles & pages \\
\hline Table S1. & Crystal Data and Structure Refinement for $\mathrm{Sn}\left(\mathrm{IO}_{3}\right)_{4}$ and $\mathrm{Sn}\left(\mathrm{IO}_{3}\right)_{2} \mathrm{~F}_{2}$. & S3 \\
\hline Table S2-S3. & $\begin{array}{l}\text { Atomic coordinates and equivalent isotropic displacement parameters for } \\
\mathrm{Sn}\left(\mathrm{IO}_{3}\right)_{4} \text { and } \mathrm{Sn}\left(\mathrm{IO}_{3}\right)_{2} \mathrm{~F}_{2} \text {, respectively. }\end{array}$ & S4-S5 \\
\hline Table S4-S5. & $\begin{array}{l}\text { Selected Bond Distances }(\AA) \text { and Angles }\left({ }^{\circ}\right) \text { for for } \mathrm{Sn}\left(\mathrm{IO}_{3}\right)_{4} \text { and } \mathrm{Sn}\left(\mathrm{IO}_{3}\right)_{2} \mathrm{~F}_{2} \text {, } \\
\text { respectively. }\end{array}$ & S4-S5 \\
\hline Table S6. & $\begin{array}{l}\text { Calculation of dipole moment for } \mathrm{IO}_{3} \text { and } \mathrm{SnO}_{4} \mathrm{~F}_{2} \text { polyhedra and net dipole } \\
\text { moment for a unit cell in } \mathrm{Sn}\left(\mathrm{IO}_{3}\right)_{2} \mathrm{~F}_{2}(\mathrm{D}=\text { Debyes })\end{array}$ & S5 \\
\hline Table S7 & $\begin{array}{l}\text { Atom-cutting analysis and calculated SHG coefficients }(\mathrm{pm} / \mathrm{V}) \text { of } \\
\mathrm{Sn}\left(\mathrm{IO}_{3}\right)_{2} \mathrm{~F}_{2}\end{array}$ & S6 \\
\hline Figure S1. & $\begin{array}{l}\text { X-ray powder diffraction patterns of }(a) \mathrm{Sn}\left(\mathrm{IO}_{3}\right)_{4} \text { and }(\mathrm{b}) \mathrm{Sn}\left(\mathrm{IO}_{3}\right)_{2} \mathrm{~F}_{2} \text {. Black } \\
\text { line is crystal sample and red line is simulation results. }\end{array}$ & S7 \\
\hline Figure S2 & $\begin{array}{l}\text { Energy dispersive X-ray spectroscopy (EDX) of (a) } \mathrm{Sn}\left(\mathrm{IO}_{3}\right)_{4} \text { and (b) } \\
\mathrm{Sn}\left(\mathrm{IO}_{3}\right)_{2} \mathrm{~F}_{2} \text {. }\end{array}$ & S8 \\
\hline Figure S3 & TGA curves of (a) $\mathrm{Sn}\left(\mathrm{IO}_{3}\right)_{4}$ and (b) $\mathrm{Sn}\left(\mathrm{IO}_{3}\right)_{2} \mathrm{~F}_{2}$. & S9 \\
\hline Figure S4 & $\begin{array}{l}\text { UV-vis-NIR diffuse-reflectance spectroscopy of (a) (a) } \mathrm{Sn}\left(\mathrm{IO}_{3}\right)_{4} \text { and (b) } \\
\mathrm{Sn}\left(\mathrm{IO}_{3}\right)_{2} \mathrm{~F}_{2} \text {. }\end{array}$ & S10 \\
\hline Figure S5 & IR spectroscopy of (a) $\mathrm{Sn}\left(\mathrm{IO}_{3}\right)_{4}$ and (b) $\mathrm{Sn}\left(\mathrm{IO}_{3}\right)_{2} \mathrm{~F}_{2}$. & $\mathrm{S} 11$ \\
\hline \multirow[t]{2}{*}{ Figure S6 } & $\begin{array}{l}\text { The band structures of (a) } \mathrm{Sn}\left(\mathrm{IO}_{3}\right)_{4} \text { and (c) } \mathrm{Sn}\left(\mathrm{IO}_{3}\right)_{2} \mathrm{~F}_{2} \text {. The partial densities of } \\
\text { states for (b) } \mathrm{Sn}\left(\mathrm{IO}_{3}\right)_{4} \text { and (d) } \mathrm{Sn}\left(\mathrm{IO}_{3}\right)_{2} \mathrm{~F}_{2} \text {. }\end{array}$ & S12 \\
\hline & $\mathrm{Sn}\left(\mathrm{IO}_{3}\right)_{2} \mathrm{~F}_{2}$ Pseudosymmetry Analysis & S13-14 \\
\hline References & & S15 \\
\hline
\end{tabular}


Table S1. Crystal Data and Structure Refinement for $\mathrm{Sn}\left(\mathrm{IO}_{3}\right)_{4}$ and $\mathrm{Sn}\left(\mathrm{IO}_{3}\right)_{2} \mathrm{~F}_{2}$

\begin{tabular}{lll}
\hline Formula & $\mathrm{Sn}\left(\mathrm{IO}_{3}\right)_{4}(\mathbf{1})$ & $\mathrm{Sn}\left(\mathrm{IO}_{3}\right)_{2} \mathrm{~F}_{2}(\mathbf{2})$ \\
\hline Formula Mass $(\mathrm{amu})$ & 818.29 & 506.49 \\
Crystal System & Triclinic & Monoclinic \\
Space Group & $P-1$ & $P 2_{1}$ \\
$\mathrm{a}(\AA)$ & $5.0576(5)$ & $5.436(13)$ \\
$\mathrm{b}(\AA)$ & $6.2808(6)$ & $9.91(2)$ \\
$\mathrm{c}(\AA)$ & $8.3865(8)$ & $6.281(15)$ \\
$\alpha$ & $87.680(2)$ & 90 \\
$\beta$ & $88.647(1)$ & $105.44(5)$ \\
$\gamma$ & $89.031(1)$ & 90 \\
$\mathrm{~V}\left(\AA^{3}\right)$ & $266.08(4)$ & $326.3(13)$ \\
$\mathrm{Z}$ & 1 & 2 \\
$\rho(\mathrm{calcd})\left(\mathrm{g} / \mathrm{cm}{ }^{3}\right)$ & 5.107 & 5.155 \\
$\mathrm{Temperature}(\mathrm{K})$ & $296(2)$ & $296(2)$ \\
$\lambda(\AA)$ & 0.71073 & 0.71073 \\
$\mathrm{~F}(000)$ & 358 & 444 \\
$\left.\mu(\mathrm{mm})^{-1}\right)$ & 14.064 & 13.404 \\
$\mathrm{R} / \mathrm{wR}(\mathrm{I}>2 \sigma(\mathrm{I}))$ & $0.0235 / 0.0544$ & $0.0328 / 0.0650$ \\
$\mathrm{R} / \mathrm{wR}($ all data $)$ & $0.0251 / 0.0552$ & $0.0359 / 0.0656$ \\
$\mathrm{GOF}$ on $\mathrm{F}^{2}$ & 1.075 & 0.905 \\
Absolute Structure Parameter & - & 0.00 \\
\hline
\end{tabular}

$\mathrm{R}(\mathrm{F})=\Sigma|| \mathrm{F}_{\mathrm{o}}|-| \mathrm{F}_{\mathrm{c}}|/ \Sigma| \mathrm{F}_{\mathrm{o}} \mid \cdot \mathrm{wR}\left(\mathrm{F}_{\mathrm{o}}^{2}\right)=\left[\Sigma \mathrm{w}\left(\mathrm{F}_{\mathrm{o}}^{2}-\mathrm{F}_{\mathrm{c}}^{2}\right)^{2} / \Sigma \mathrm{w}\left(\mathrm{F}_{\mathrm{o}}{ }^{2}\right)^{2}\right.$

Table S2. Atomic Coordinates and Equivalent Isotropic Displacement Parameters for

\begin{tabular}{llllll}
\multicolumn{5}{c}{$\mathrm{Sn}\left(\mathrm{IO}_{3}\right)_{4}$} \\
$\mathrm{Sn}(1)$ & $\mathrm{x}$ & $\mathrm{y}$ & $\mathrm{z}$ & $\mathrm{U}(\mathrm{eq})^{\mathrm{a}}$ & $\mathrm{BVS}$ \\
$\mathrm{I}(1)$ & 10000 & 10000 & 5000 & $7(1)$ & 4.07 \\
$\mathrm{I}(2)$ & $5055(1)$ & $7720(1)$ & $2462(1)$ & $8(1)$ & 4.93 \\
$\mathrm{O}(1)$ & $9787(1)$ & $7048(1)$ & $8461(1)$ & $9(1)$ & 4.87 \\
$\mathrm{O}(2)$ & $7725(8)$ & $9500(6)$ & $3063(4)$ & $15(1)$ & 2.14 \\
$\mathrm{O}(3)$ & $2928(8)$ & $7940(7)$ & $4239(5)$ & $16(1)$ & 2.26 \\
$\mathrm{O}(4)$ & $8517(8)$ & $7577(6)$ & $6424(5)$ & $13(1)$ & 2.18 \\
$\mathrm{O}(5)$ & $8330(8)$ & $4441(6)$ & $8807(5)$ & $16(1)$ & 2.09 \\
$\mathrm{O}(6)$ & $6611(8)$ & $5254(7)$ & $2983(5)$ & $19(1)$ & 2.06 \\
\hline
\end{tabular}

${ }^{\mathrm{a}} \mathrm{U}_{\mathrm{eq}}$ is defined as one third of the trace of the orthogonalized $\mathrm{U}_{\mathrm{ij}}$ tensor. 
Table S3. Atomic Coordinates and Equivalent Isotropic Displacement Parameters for

\begin{tabular}{llllll}
\multicolumn{5}{c}{$\mathrm{Sn}\left(\mathrm{IO}_{3}\right)_{2} \mathrm{~F}_{2}$} \\
$\mathrm{Sn}(1)$ & $\mathrm{x}$ & $\mathrm{y}$ & $\mathrm{z}$ & $\mathrm{U}(\mathrm{eq})^{\mathrm{a}}$ & $\mathrm{BVS}$ \\
$\mathrm{I}(1)$ & $-1947(2)$ & $1287(1)$ & $7124(2)$ & $12(1)$ & 4.16 \\
$\mathrm{I}(2)$ & $4173(2)$ & $2446(1)$ & $545(1)$ & $13(1)$ & 4.87 \\
$\mathrm{O}(1)$ & $1921(2)$ & $204(1)$ & $3663(1)$ & $13(1)$ & 4.87 \\
$\mathrm{O}(2)$ & $-485(16)$ & $33(11)$ & $5218(14)$ & $11(2)$ & 2.27 \\
$\mathrm{O}(3)$ & $1385(18)$ & $1950(9)$ & $2924(15)$ & $15(2)$ & 2.04 \\
$\mathrm{O}(4)$ & $1386(16)$ & $2376(10)$ & $-1822(14)$ & $15(2)$ & 2.28 \\
$\mathrm{O}(5)$ & $6515(18)$ & $2632(11)$ & $-1102(16)$ & $21(2)$ & 2.16 \\
$\mathrm{O}(6)$ & $4662(16)$ & $268(10)$ & $6099(13)$ & $16(2)$ & 2.25 \\
$\mathrm{~F}(1)$ & $4762(18)$ & $695(9)$ & $1213(16)$ & $19(2)$ & 2.00 \\
$\mathrm{~F}(2)$ & $-2881(13)$ & $2631(8)$ & $-4735(11)$ & $17(2)$ & 0.92 \\
\hline
\end{tabular}

${ }^{\mathrm{a}} \mathrm{U}_{\mathrm{eq}}$ is defined as one third of the trace of the orthogonalized $\mathrm{U}_{\mathrm{ij}}$ tensor.

Table S4. Selected Bond Distances $(\AA)$ and Angles $\left({ }^{\circ}\right)$ for $\mathrm{Sn}\left(\mathrm{IO}_{3}\right)_{4}$

\begin{tabular}{|c|c|c|c|}
\hline $\mathrm{I}(1)-\mathrm{O}(5)$ & $1.769(4)$ & $\mathrm{Sn}(1)-\mathrm{O}(3) \# 2$ & $2.038(4)$ \\
\hline $\mathrm{I}(1)-\mathrm{O}(2)$ & $1.826(4)$ & $\mathrm{Sn}(1)-\mathrm{O}(1)$ & $2.051(4)$ \\
\hline $\mathrm{I}(1)-\mathrm{O}(1)$ & $1.859(4)$ & $\operatorname{Sn}(1)-O(1) \# 2$ & $2.051(4)$ \\
\hline I(1)-O(4)\#1 & $2.487(4)$ & $\mathrm{Sn}(1)-\mathrm{O}(2) \# 3$ & $2.057(4)$ \\
\hline$I(2)-O(6)$ & $1.793(4)$ & $\operatorname{Sn}(1)-O(2) \# 4$ & $2.057(4)$ \\
\hline $\mathrm{I}(2)-\mathrm{O}(4)$ & $1.817(4)$ & $O(2)-S n(1) \# 5$ & $2.057(4)$ \\
\hline $\mathrm{I}(2)-\mathrm{O}(3)$ & $1.855(4)$ & O(4)-I(1)\#1 & $2.487(4)$ \\
\hline $\mathrm{Sn}(1)-\mathrm{O}(3)$ & $2.038(4)$ & & \\
\hline$O(5)-I(1)-O(2)$ & $98.07(18)$ & $O(3)-S n(1)-O(2) \# 3$ & $88.57(16)$ \\
\hline$O(5)-I(1)-O(1)$ & $98.01(19)$ & $\mathrm{O}(3) \# 2-\mathrm{Sn}(1)-\mathrm{O}(2) \# 3$ & $91.43(16)$ \\
\hline $\mathrm{O}(2)-\mathrm{I}(1)-\mathrm{O}(1)$ & $97.80(18)$ & $\mathrm{O}(1)-\mathrm{Sn}(1)-\mathrm{O}(2) \# 3$ & $92.59(16)$ \\
\hline $\mathrm{O}(5)-\mathrm{I}(1)-\mathrm{O}(4) \# 1$ & $85.79(17)$ & $\mathrm{O}(1) \# 2-\mathrm{Sn}(1)-\mathrm{O}(2) \# 3$ & $87.41(16)$ \\
\hline$O(2)-I(1)-O(4) \# 1$ & $90.41(17)$ & $O(3)-S n(1)-O(2) \# 4$ & $91.43(16)$ \\
\hline $\mathrm{O}(1)-\mathrm{I}(1)-\mathrm{O}(4) \# 1$ & $170.35(15)$ & $\mathrm{O}(3) \# 2-\mathrm{Sn}(1)-\mathrm{O}(2) \# 4$ & $88.57(16)$ \\
\hline$O(6)-I(2)-O(4)$ & $99.6(2)$ & $\mathrm{O}(1)-\mathrm{Sn}(1)-\mathrm{O}(2) \# 4$ & $87.41(16)$ \\
\hline$O(6)-1(2)-O(3)$ & $96.9(2)$ & $\mathrm{O}(1) \# 2-\mathrm{Sn}(1)-\mathrm{O}(2) \# 4$ & $92.59(16)$ \\
\hline$O(4)-1(2)-O(3)$ & $97.00(17)$ & $\mathrm{O}(2) \# 3-\mathrm{Sn}(1)-\mathrm{O}(2) \# 4$ & $180.000(1)$ \\
\hline $\mathrm{O}(3)-\mathrm{Sn}(1)-\mathrm{O}(3) \# 2$ & $180.00(15)$ & $\mathrm{I}(1)-\mathrm{O}(1)-\mathrm{Sn}(1)$ & $139.5(2)$ \\
\hline$O(3)-S n(1)-O(1)$ & $96.65(15)$ & $\mathrm{I}(1)-\mathrm{O}(2)-\mathrm{Sn}(1) \# 5$ & $136.1(2)$ \\
\hline $\mathrm{O}(3) \# 2-\mathrm{Sn}(1)-\mathrm{O}(1)$ & $83.35(15)$ & $\mathrm{I}(2)-O(3)-S n(1)$ & $120.5(2)$ \\
\hline $\mathrm{O}(3)-\mathrm{Sn}(1)-\mathrm{O}(1) \# 2$ & $83.35(15)$ & $\mathrm{I}(2)-\mathrm{O}(4)-\mathrm{I}(1) \# 1$ & $136.9(2)$ \\
\hline $\mathrm{O}(3) \# 2-\mathrm{Sn}(1)-\mathrm{O}(1) \# 2$ & $96.65(15)$ & & \\
\hline
\end{tabular}

Symmetry transformations used to generate equivalent atoms:

$$
\# 1-x+1,-y+1,-z+1 \quad \# 2-x+2,-y+2,-z+1
$$


\#3 $\mathrm{x}+1, \mathrm{y}, \mathrm{z} \quad$ \#4 - $\mathrm{x}+1,-\mathrm{y}+2,-\mathrm{z}+1 \quad$ \#5 $\mathrm{x}-1, \mathrm{y}, \mathrm{z}$

Table S5. Selected Bond Distances $(\AA)$ and Angles $\left({ }^{\circ}\right)$ for $\mathrm{Sn}\left(\mathrm{IO}_{3}\right)_{2} \mathrm{~F}_{2}$

\begin{tabular}{|c|c|c|c|}
\hline $\mathrm{I}(1)-\mathrm{O}(6)$ & $1.795(10)$ & $S n(1)-F(1)$ & $1.970(8)$ \\
\hline $\mathrm{I}(1)-\mathrm{O}(3)$ & $1.820(9)$ & $\operatorname{Sn}(1)-O(1)$ & $2.028(9)$ \\
\hline $\mathrm{I}(1)-\mathrm{O}(4)$ & $1.851(9)$ & $\operatorname{Sn}(1)-O(5) \# 1$ & $2.049(10)$ \\
\hline $\mathrm{I}(1)-\mathrm{O}(2)$ & $2.445(10)$ & $\operatorname{Sn}(1)-O(4) \# 2$ & $2.054(10)$ \\
\hline $\mathrm{I}(2)-\mathrm{O}(2)$ & $1.797(10)$ & $\mathrm{Sn}(1)-\mathrm{O}(3) \# 3$ & $2.061(10)$ \\
\hline$I(2)-O(5)$ & $1.831(9)$ & $O(3)-S n(1) \# 4$ & $2.061(10)$ \\
\hline $\mathrm{I}(2)-\mathrm{O}(1)$ & $1.837(9)$ & $O(4)-S n(1) \# 5$ & $2.054(10)$ \\
\hline $\operatorname{Sn}(1)-F(2)$ & $1.953(8)$ & $O(5)-S n(1) \# 6$ & $2.049(10)$ \\
\hline$O(6)-I(1)-O(3)$ & $102.3(5)$ & $F(2)-S n(1)-O(4) \# 2$ & $94.5(4)$ \\
\hline$O(6)-I(1)-O(4)$ & $96.9(5)$ & $F(1)-S n(1)-O(4) \# 2$ & $85.1(4)$ \\
\hline$O(3)-I(1)-O(4)$ & $95.4(4)$ & $\mathrm{O}(1)-\mathrm{Sn}(1)-\mathrm{O}(4) \# 2$ & $176.6(5)$ \\
\hline$O(6)-I(1)-O(2)$ & $76.3(4)$ & $\mathrm{O}(5) \# 1-\mathrm{Sn}(1)-\mathrm{O}(4) \# 2$ & $91.6(4)$ \\
\hline$O(3)-I(1)-O(2)$ & $88.6(4)$ & $F(2)-S n(1)-O(3) \# 3$ & $94.4(4)$ \\
\hline$O(4)-I(1)-O(2)$ & $172.8(4)$ & $F(1)-S n(1)-O(3) \# 3$ & $85.2(3)$ \\
\hline$O(2)-I(2)-O(5)$ & $102.8(5)$ & $O(1)-S n(1)-O(3) \# 3$ & $93.6(4)$ \\
\hline$O(2)-I(2)-O(1)$ & $97.9(4)$ & $\mathrm{O}(5) \# 1-\mathrm{Sn}(1)-\mathrm{O}(3) \# 3$ & $177.7(4)$ \\
\hline$O(5)-I(2)-O(1)$ & $95.5(4)$ & $\mathrm{O}(4) \# 2-\mathrm{Sn}(1)-\mathrm{O}(3) \# 3$ & $86.3(4)$ \\
\hline$F(2)-S n(1)-F(1)$ & $179.4(4)$ & $\mathrm{I}(2)-O(1)-\mathrm{Sn}(1)$ & $134.7(6)$ \\
\hline$F(2)-S n(1)-O(1)$ & $88.9(4)$ & $I(2)-O(2)-I(1)$ & $105.5(4)$ \\
\hline$F(1)-S n(1)-O(1)$ & $91.6(4)$ & $\mathrm{I}(1)-\mathrm{O}(3)-\mathrm{Sn}(1) \# 4$ & $138.7(5)$ \\
\hline$F(2)-S n(1)-O(5) \# 1$ & $86.5(3)$ & $\mathrm{I}(1)-\mathrm{O}(4)-\mathrm{Sn}(1) \# 5$ & $132.7(6)$ \\
\hline$F(1)-S n(1)-O(5) \# 1$ & $93.8(4)$ & $\mathrm{I}(2)-O(5)-\mathrm{Sn}(1) \# 6$ & $138.0(5)$ \\
\hline$O(1)-S n(1)-O(5) \# 1$ & $88.5(4)$ & & \\
\hline
\end{tabular}

Symmetry transformations used to generate equivalent atoms:

$$
\begin{array}{lll}
\# 1 \mathrm{x}-1, \mathrm{y}, \mathrm{z} & \# 2 \mathrm{x}-1, \mathrm{y}, \mathrm{z}+1 & \# 3 \mathrm{x}, \mathrm{y}, \mathrm{z}+1 \\
\# 4 \mathrm{x}, \mathrm{y}, \mathrm{z}-1 & \# 5 \mathrm{x}+1, \mathrm{y}, \mathrm{z}-1 & \# 6 \mathrm{x}+1, \mathrm{y}, \mathrm{z}
\end{array}
$$

Table S6. Calculation of dipole moment for $\mathrm{IO}_{3}$ and $\mathrm{SnO}_{4} \mathrm{~F}_{2}$ polyhedra and net dipole moment for a unit cell in $\mathrm{Sn}\left(\mathrm{IO}_{3}\right)_{2} \mathrm{~F}_{2}(\mathrm{D}=$ Debyes $)$.

\begin{tabular}{ccccc}
\hline species & x-component & $y$-component & z-component & $\begin{array}{c}\text { Total } \\
\text { magnitude }\end{array}$ \\
\hline $\mathrm{IO}_{3}$ & -3.344349836 & -9.448176828 & 9.89748988 & 14.08591591 \\
& -2.812348421 & 9.627494318 & 10.34331056 & 14.4077071 \\
& 2.813679619 & 9.625593509 & -10.34499283 & 14.40790477 \\
& 3.344444677 & -9.448219186 & -9.89770582 & 14.08611857 \\
& $U_{x}$ & $U_{y}$ & $U_{z}$ & $U_{t}$ \\
& 0.001426039 & 0.356692 & -0.0018982 & 0.356699716 \\
\hline & & & & \\
$\mathrm{SnO}_{4} \mathrm{~F}_{2}$ & 0.222141 & -0.538177739 & 0.428746746 & 0.723053023 \\
& -0.221579048 & -0.537607733 & -0.426360905 & 0.721042974 \\
& $U_{x}$ & $U_{y}$ & $U_{z}$ & $U_{t}$ \\
\hline
\end{tabular}




\begin{tabular}{ccccc}
\hline \multicolumn{6}{c}{0.000561952} & -1.075785472 & 0.002385842 & 1.075788264 \\
\hline \multicolumn{5}{c}{ Table S7 Calculated SHG coefficients and atom-cutting analysis of $\mathrm{Sn}\left(\mathrm{IO}_{3}\right)_{2} \mathrm{~F}_{2}$} \\
\hline & $d_{14}$ & $d_{16}$ & $d_{22}$ & $d_{23}$ \\
& $(\mathrm{pm} / \mathrm{V})$ & $(\mathrm{pm} / \mathrm{V})$ & $(\mathrm{pm} / \mathrm{V})$ & $(\mathrm{pm} / \mathrm{V})$ \\
\hline Orginal & -0.12 & -0.35 & $\mathbf{- 1 . 1 3}$ & -0.09 \\
$\left(\mathrm{IO}_{3}\right)^{-}$ & -0.20 & -0.28 & $\mathbf{- 0 . 6 6}$ & 0.17 \\
$\left(\mathrm{SnO}_{4} \mathrm{~F}_{2}\right)^{6-}$ & -0.06 & -0.16 & $\mathbf{- 0 . 7 2}$ & -0.32 \\
\hline
\end{tabular}




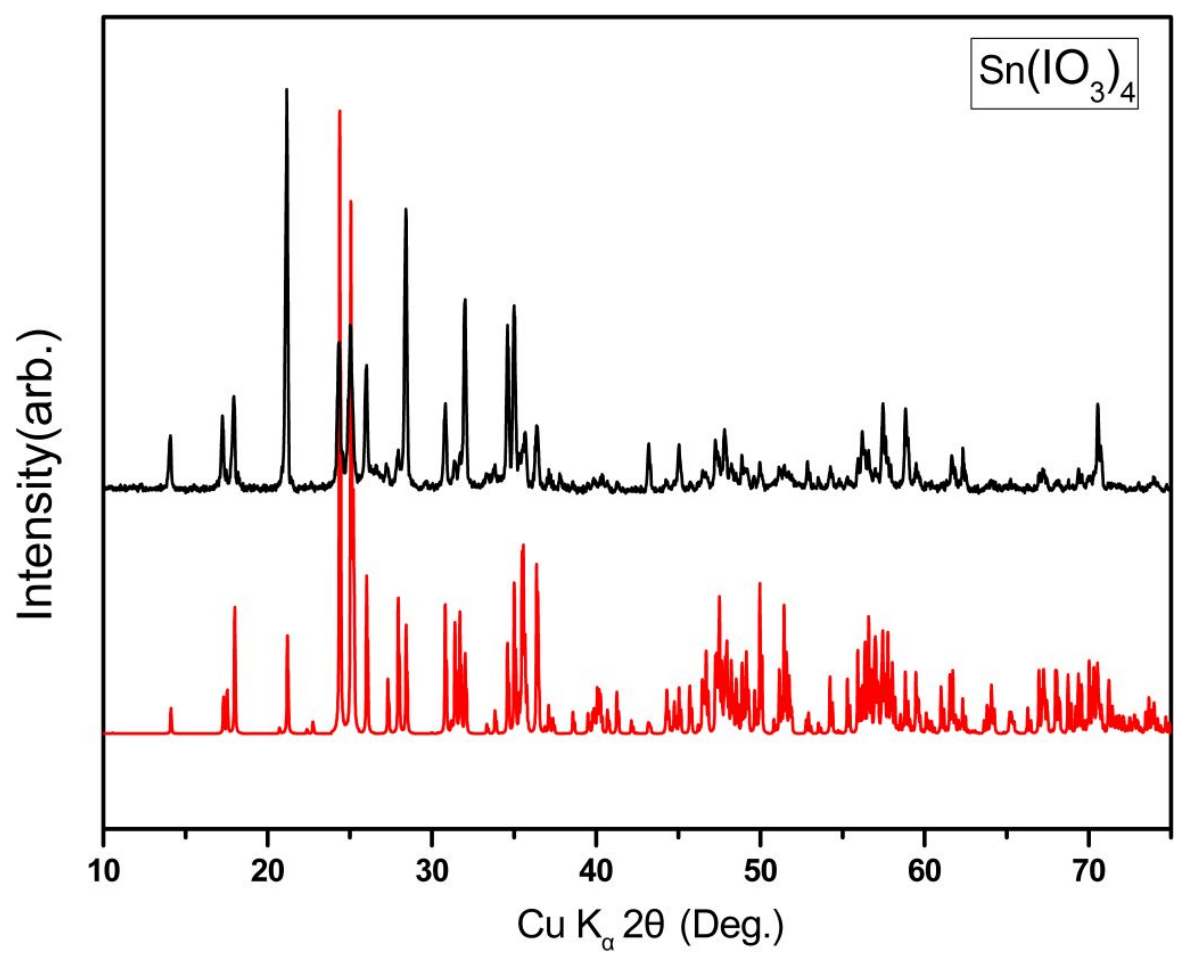

(a)

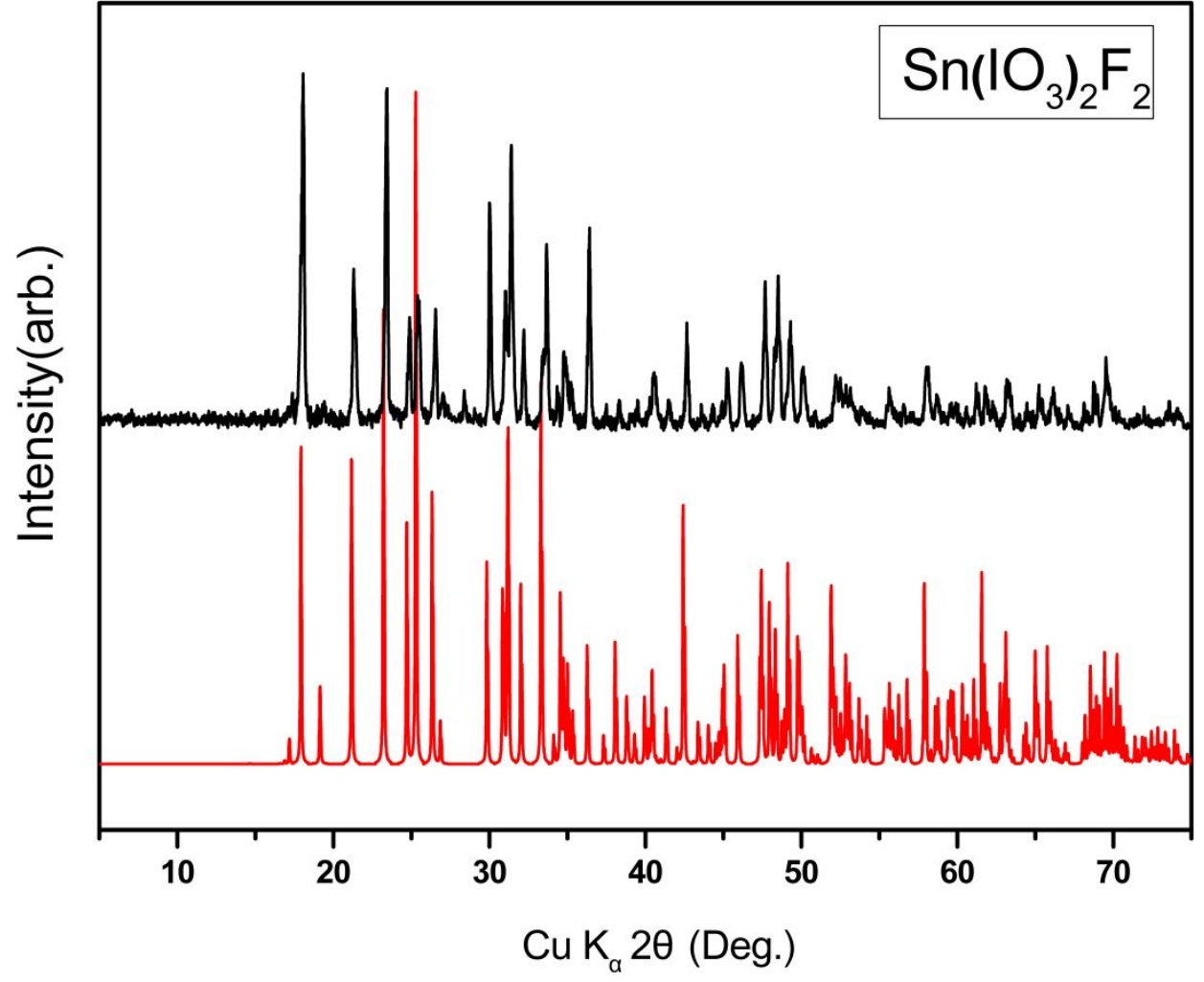

(b)

Figure S1 X-ray powder diffraction patterns of (a) $\mathrm{Sn}\left(\mathrm{IO}_{3}\right)_{4}$ and (b) $\mathrm{Sn}\left(\mathrm{IO}_{3}\right)_{2} \mathrm{~F}_{2}$. Black line is crystal sample and red line is simulation results. 


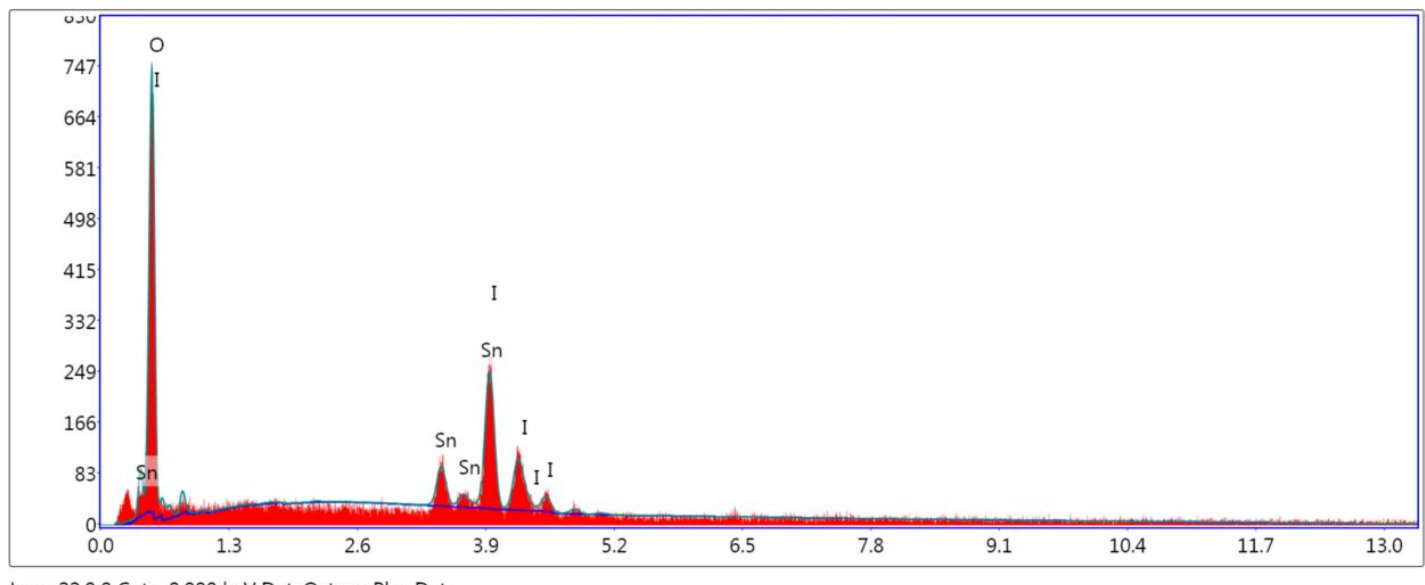

(a)

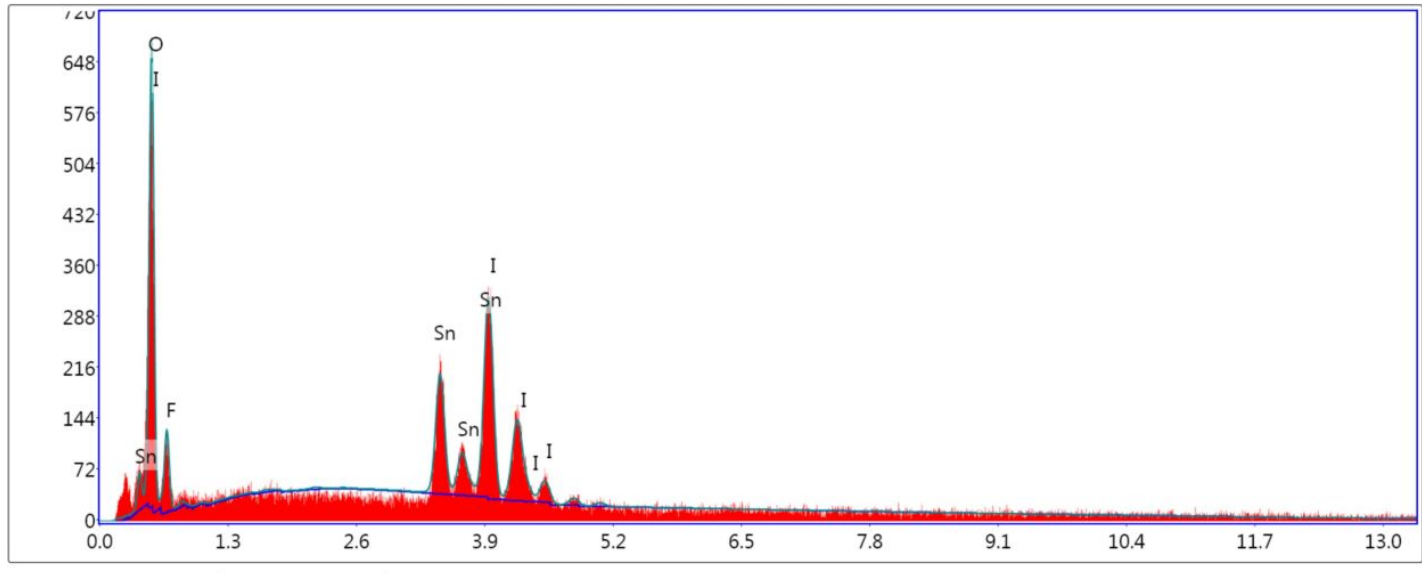

(b)

Figure S2 Energy dispersive X-ray spectroscopy (EDX) of (a) $\mathrm{Sn}\left(\mathrm{IO}_{3}\right)_{4}$ and (b) $\mathrm{Sn}\left(\mathrm{IO}_{3}\right)_{2} \mathrm{~F}_{2}$. Energy-dispersive X-ray spectroscopy (EDS) analysis revealed that the existence of $\mathrm{Sn}, \mathrm{I}, \mathrm{O}$ in $\mathbf{1}$ and $\mathrm{Sn}, \mathrm{I}, \mathrm{O}, \mathrm{F}$ in 2 


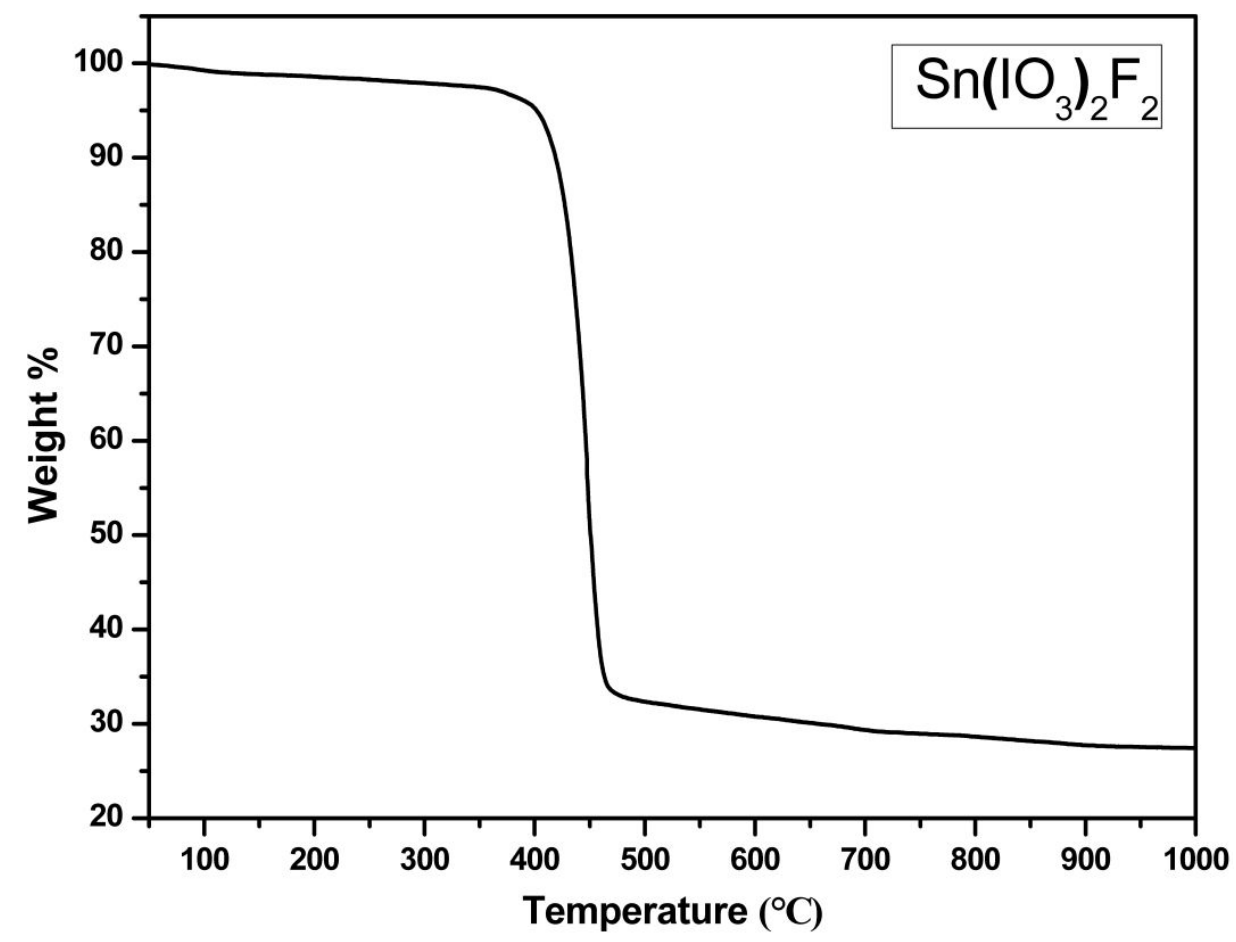

(a)

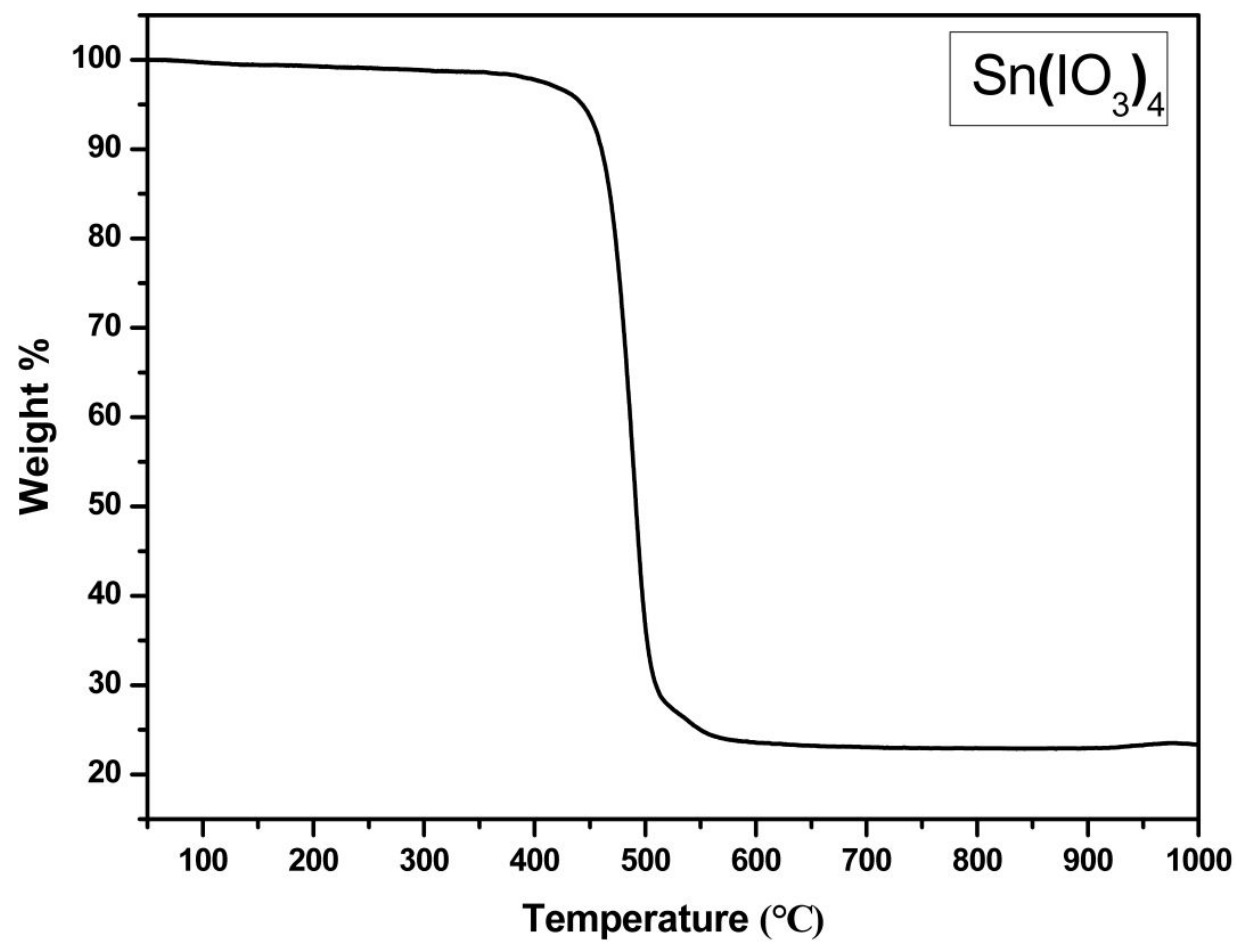

(b)

Figure S3 TGA curve of (a) $\mathrm{Sn}\left(\mathrm{IO}_{3}\right)_{4}$ and (b) $\mathrm{Sn}\left(\mathrm{IO}_{3}\right)_{2} \mathrm{~F}_{2}$

Thermal analysis indicates the stability of $\mathrm{Sn}\left(\mathrm{IO}_{3}\right)_{4}$ and $\mathrm{Sn}\left(\mathrm{IO}_{3}\right)_{2} \mathrm{~F}_{2}$ below $450^{\circ} \mathrm{C}$ and $400^{\circ} \mathrm{C}$, respectively. 


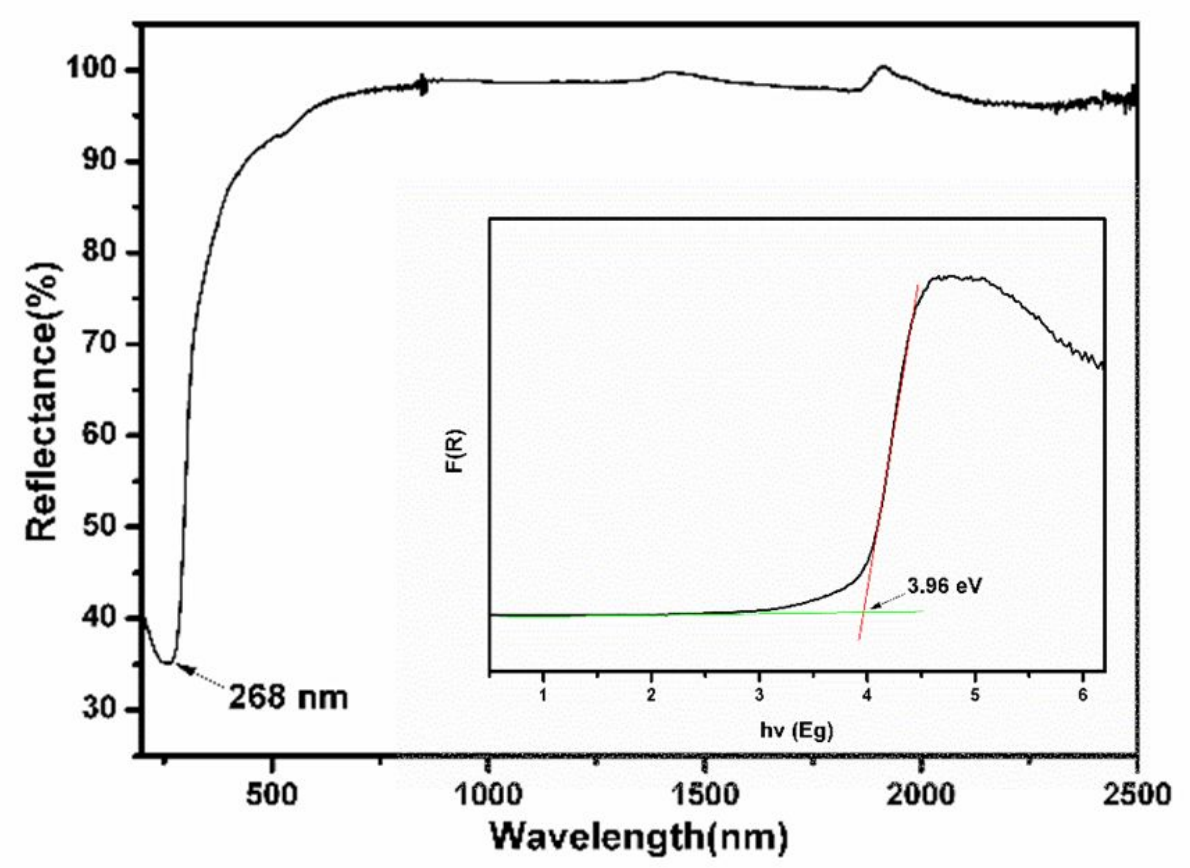

(a)

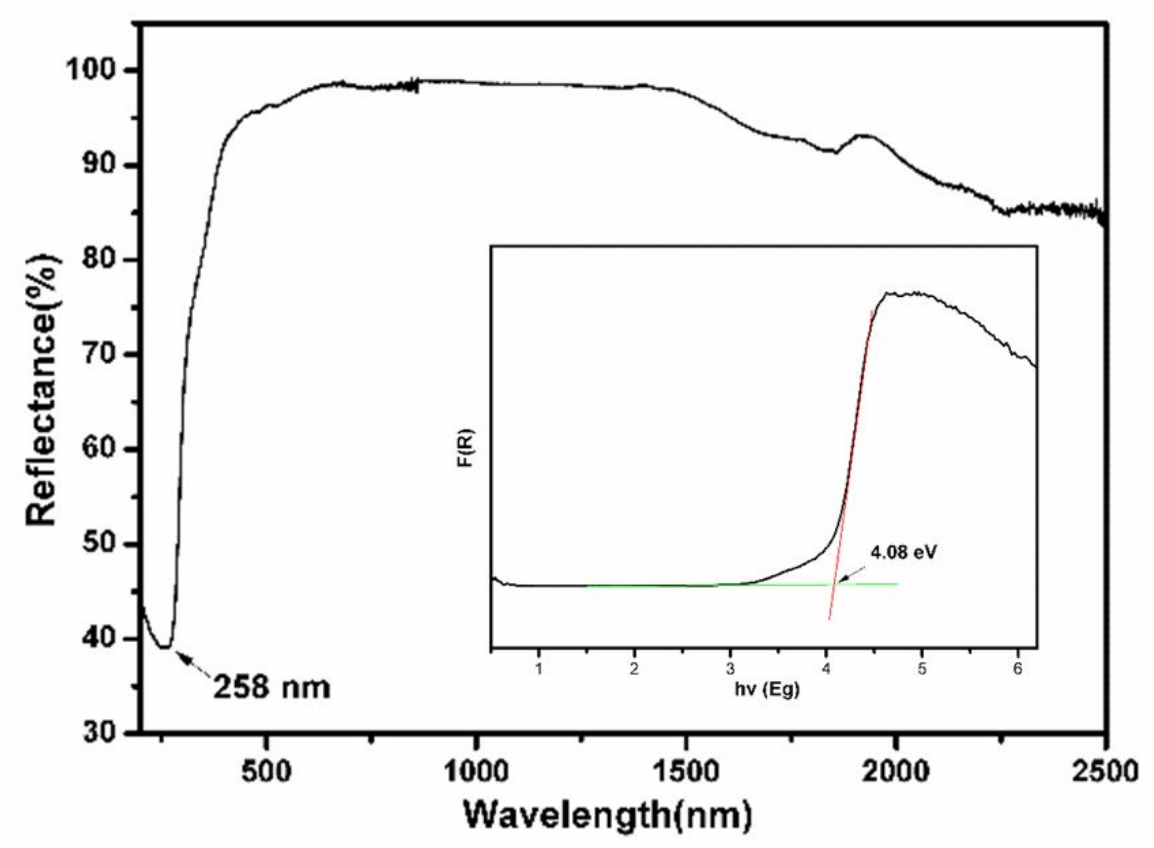

(b)

Figure S4 UV-vis-NIR diffuse-reflectance spectroscopy of (a) $\mathrm{Sn}\left(\mathrm{IO}_{3}\right)_{4}$ and (b) $\mathrm{Sn}\left(\mathrm{IO}_{3}\right)_{2} \mathrm{~F}_{2}$. 


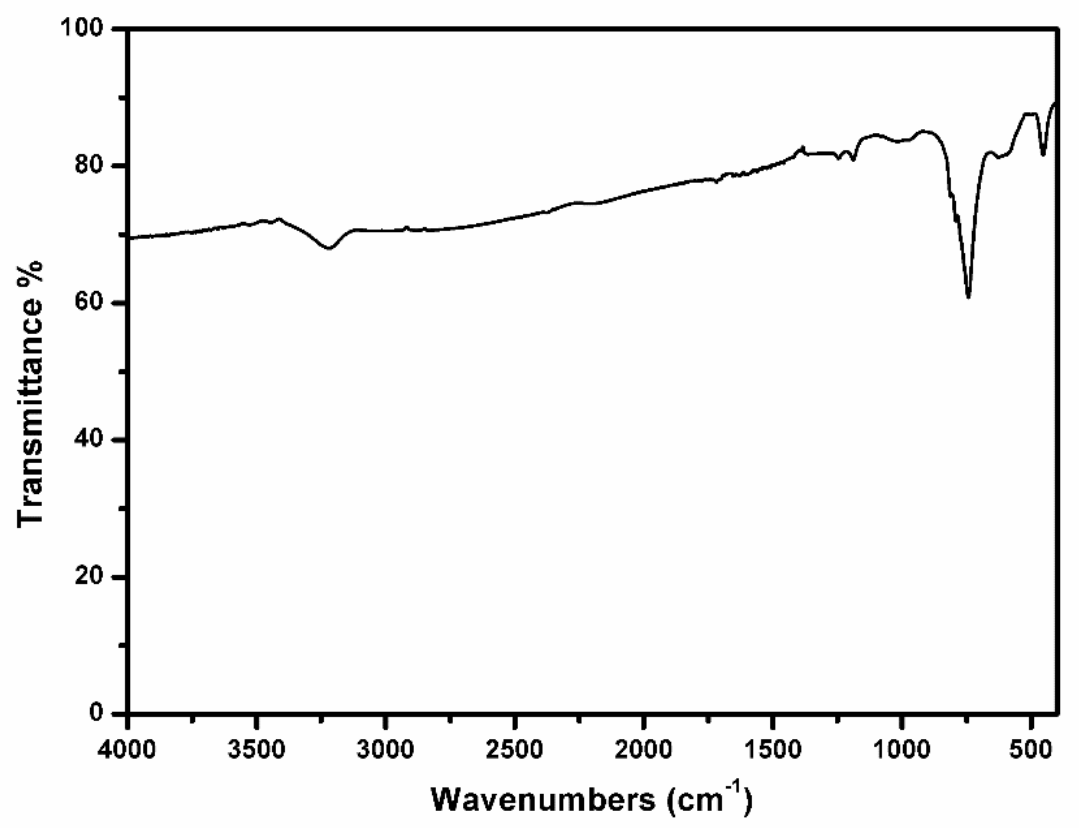

(a)

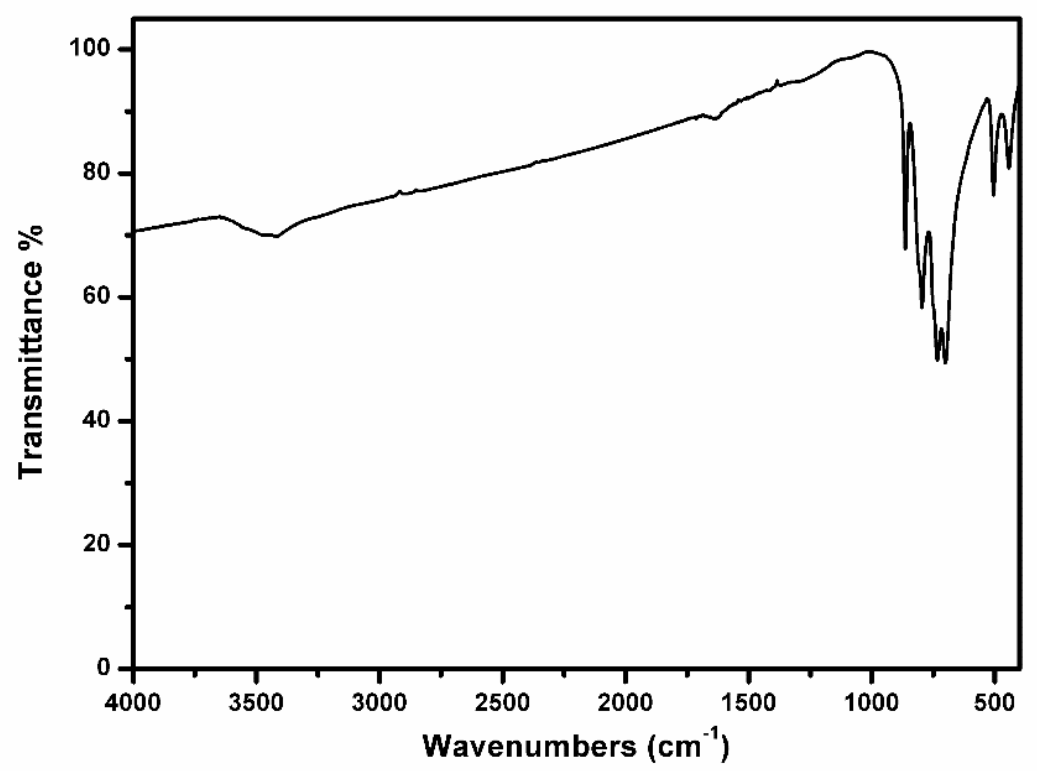

(b)

Figure S5 IR spectroscopy of (a) $\mathrm{Sn}\left(\mathrm{IO}_{3}\right)_{4}$ and (b) $\mathrm{Sn}\left(\mathrm{IO}_{3}\right)_{2} \mathrm{~F}_{2}$. 
(a)

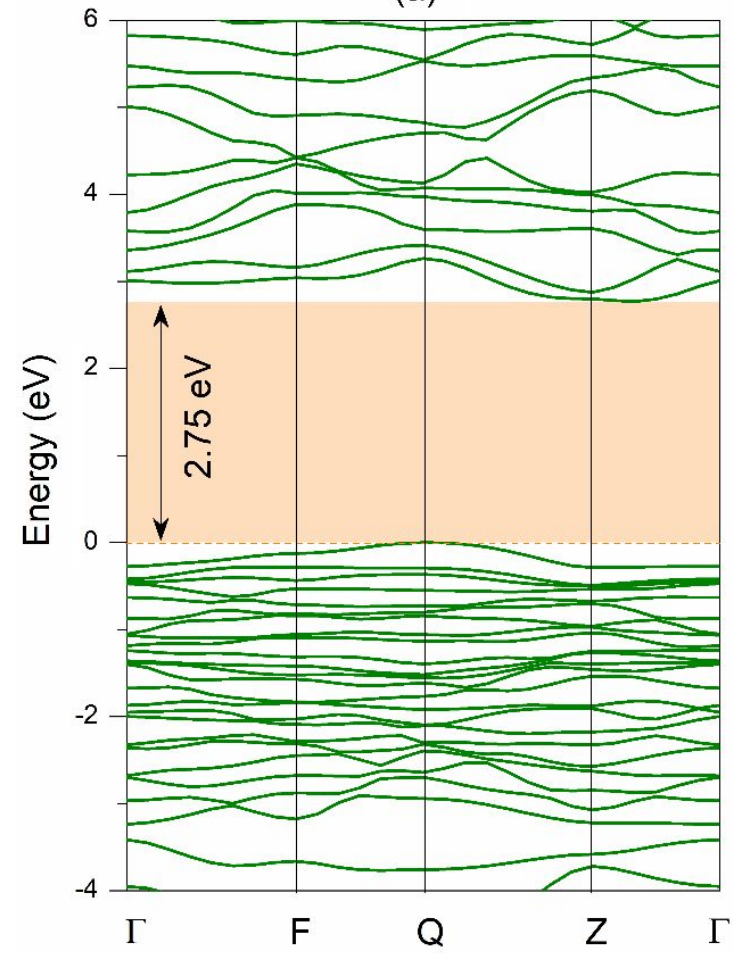

(c)

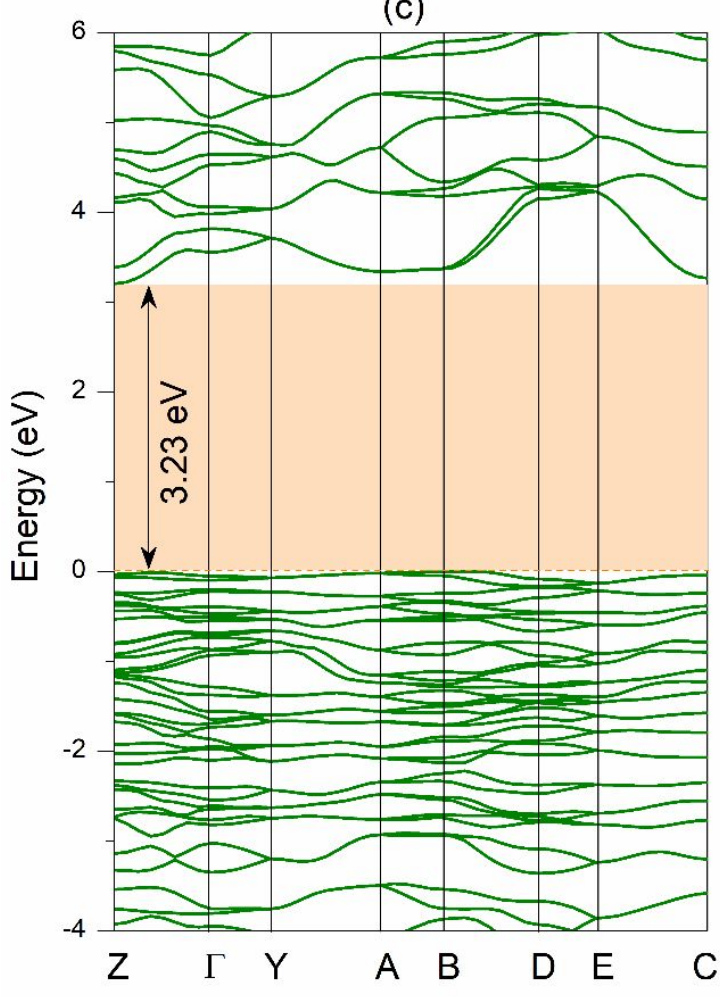

(b)

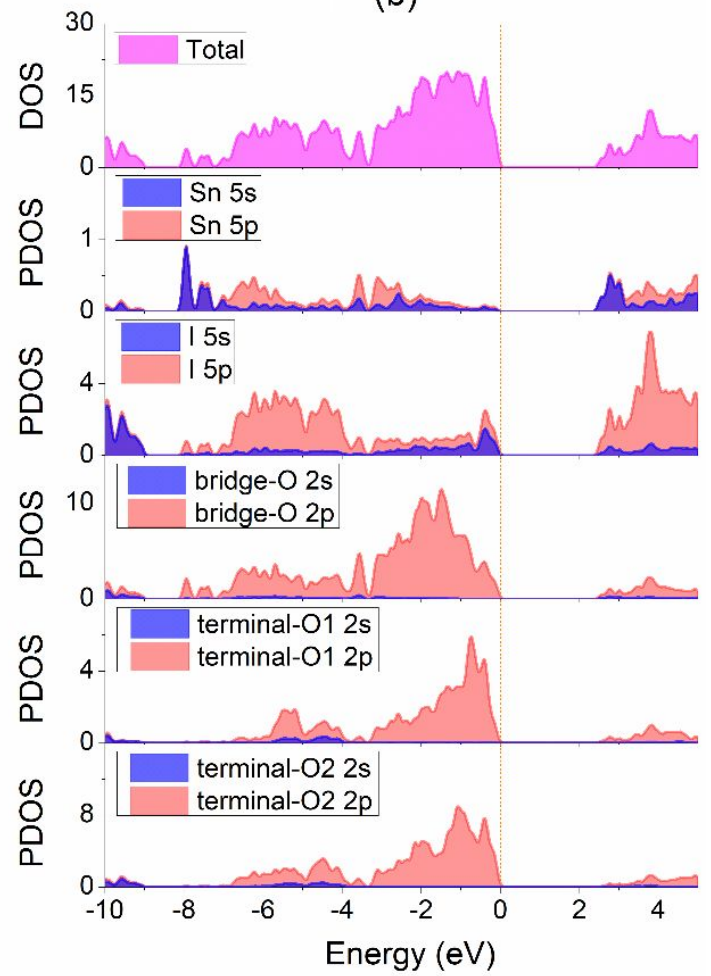

(d)

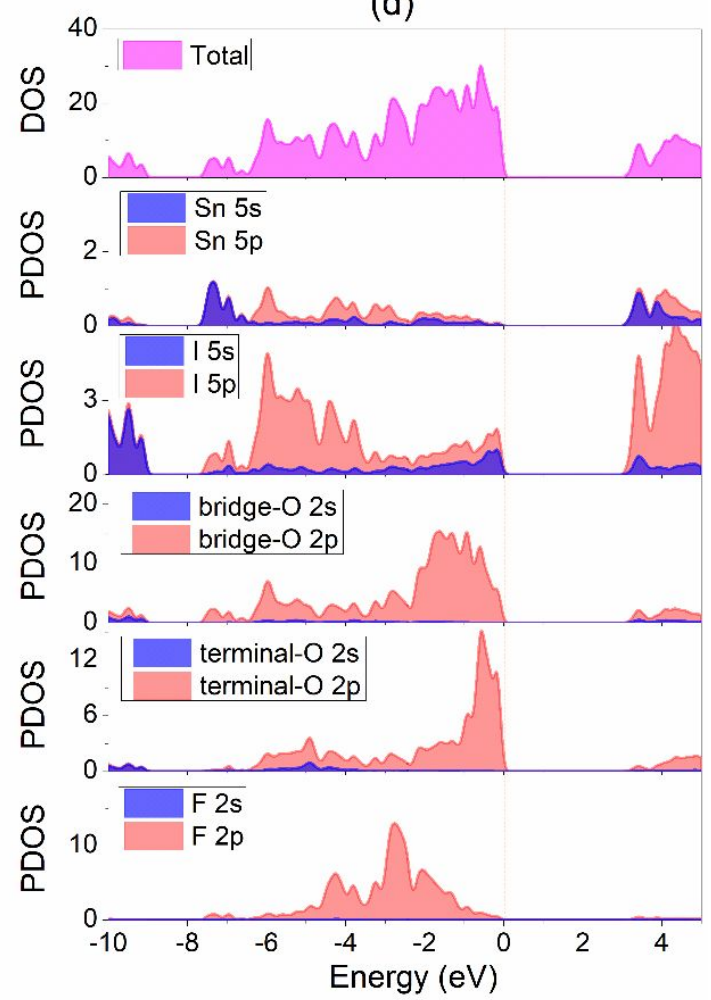

Figure S6. The band structures of (a) $\mathrm{Sn}\left(\mathrm{IO}_{3}\right)_{4}$ and (c) $\mathrm{Sn}\left(\mathrm{IO}_{3}\right)_{2} \mathrm{~F}_{2}$. The partial densities of states for (b) $\mathrm{Sn}\left(\mathrm{IO}_{3}\right)_{4}$ and (d) $\mathrm{Sn}\left(\mathrm{IO}_{3}\right)_{2} \mathrm{~F}_{2}$. 


\section{$\mathrm{Sn}\left(\mathrm{IO}_{3}\right)_{2} \mathrm{~F}_{2}$ Pseudosymmetry Analysis}

$\mathbf{S n}\left(\mathbf{I O}_{3}\right)_{2} \mathbf{F}_{2}$ forms in a polar space group P21 (Figure S6a), its pseudosymmetric centric structure has space group symmetry $P 21 / c$ (Figure S6b). We obtain the pseudosymmetric space group using the Pseudo software ${ }^{1}$ available on the Bilbao Crystallographic server ${ }^{2}$. The NCS structures are related to the CS phase by the transformation of two displacive modes described by the irreducible representations (irrep). In general, the first mode, represented by irrep $\Gamma_{1}^{+}$, contains fully symmetric and regular displacements of all the atoms, and does not result in any change in symmetry. The second mode, given by irrep $\Gamma_{1}^{-}$, involves displacements of the Sn1, I1, 01, 02, 03, and F1 atoms, which mediates the loss of inversion symmetry. In addition, the displacive mode amplitudes were calculated (Table S8).

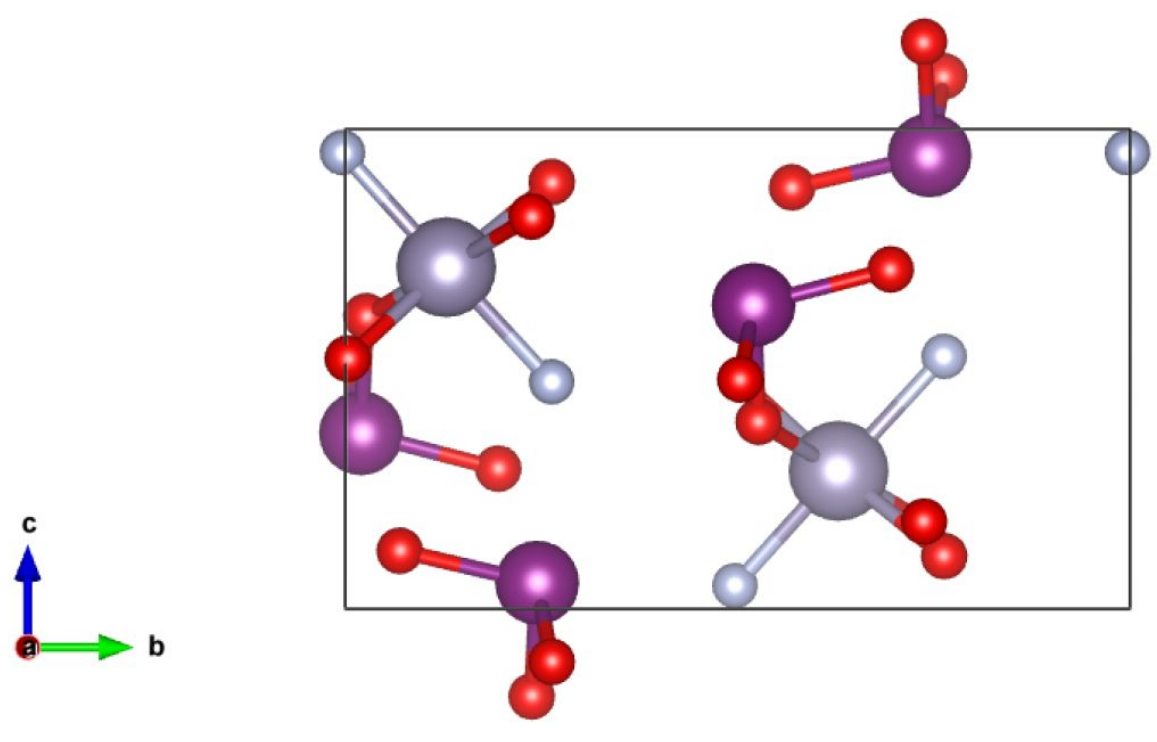

(a)

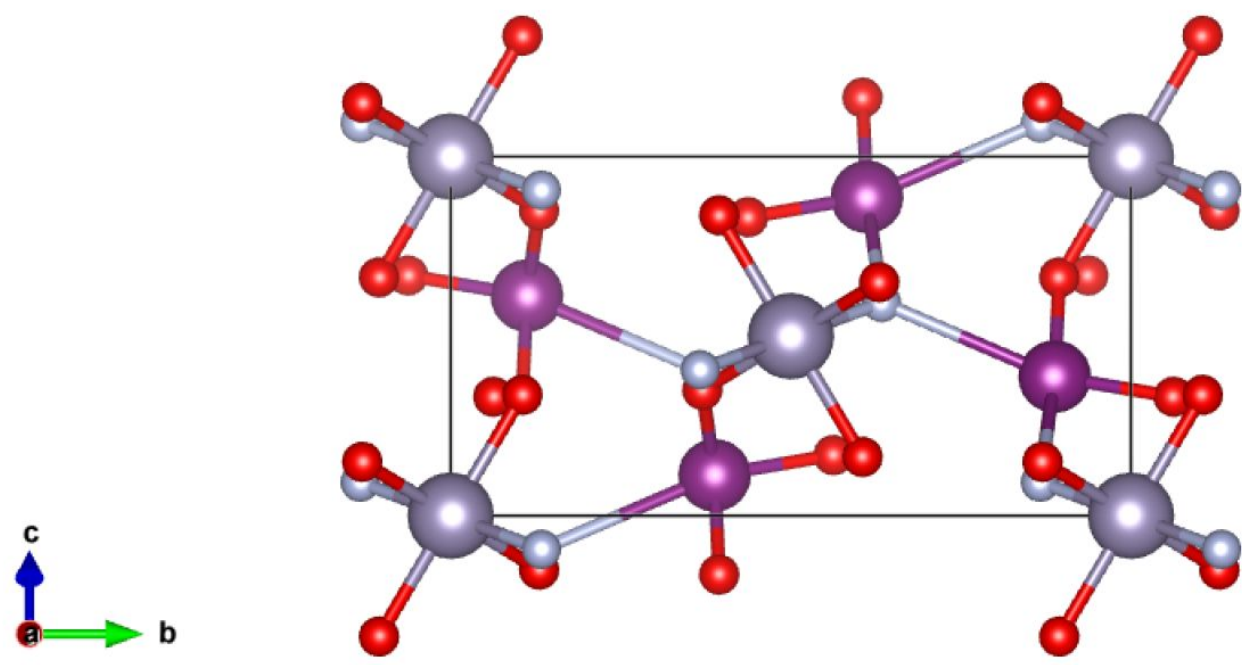

(b)

Figure S6 (a)Subgroup Distorted superstructure P21; (b) Pseudosymmetry: Parent structure (P21/c) 
Table S8. The calculated displacive mode amplitudes.

\begin{tabular}{|c|c|c|}
\hline Mode details & Amplitude & $\mathrm{d}_{\max }$ \\
\hline$[0,0,0]$ GM1-[I1:e:dsp]A_1(a) & 0.02333 & 0.01167 \\
\hline$[0,0,0]$ GM1-[I1:e:dsp]A_2(a) & -0.87099 & 0.43550 \\
\hline$[0,0,0] \mathrm{GM} 1-[\mathrm{I1}: \mathrm{e}: \mathrm{dsp}] \mathrm{A} \_3(\mathrm{a})$ & 0.00000 & 0.00000 \\
\hline$[0,0,0] \mathrm{GM} 1-[\mathrm{Sn} 1: \mathrm{a}: \mathrm{dsp}] \mathrm{Au} \_1(\mathrm{a})$ & -0.00217 & 0.00153 \\
\hline$[0,0,0]$ GM1-[Sn1:a:dsp]Au_2(a) & 0.60675 & 0.42904 \\
\hline$[0,0,0] \mathrm{GM} 1-[\mathrm{Sn} 1: \mathrm{a}: \mathrm{dsp}] \mathrm{Au} \_3(\mathrm{a})$ & -0.05326 & 0.03766 \\
\hline$[0,0,0]$ GM1-[O1:e:dsp]A_1(a) & 0.08084 & 0.04042 \\
\hline$[0,0,0]$ GM1-[O1:e:dsp]A_2(a) & -0.88364 & 0.44182 \\
\hline$[0,0,0]$ GM1-[O1:e:dsp]A_3(a) & 0.01586 & 0.00793 \\
\hline$[0,0,0]$ GM1-[O2:e:dsp]A_1(a) & 0.03832 & 0.01916 \\
\hline$[0,0,0]$ GM1-[O2:e:dsp]A_2(a) & -0.92797 & 0.46399 \\
\hline$[0,0,0]$ GM1-[O2:e:dsp]A_3(a) & -0.00396 & 0.00198 \\
\hline$[0,0,0]$ GM1-[F1:e:dsp]A_1(a) & 0.00224 & 0.00112 \\
\hline$[0,0,0] \mathrm{GM} 1-[\mathrm{F} 1: \mathrm{e}: \mathrm{dsp}] \mathrm{A} \_2(\mathrm{a})$ & -0.85871 & 0.42936 \\
\hline$[0,0,0] \mathrm{GM} 1-[\mathrm{F} 1: \mathrm{e}: \mathrm{dsp}] \mathrm{A} \_3(\mathrm{a})$ & -0.05054 & 0.02527 \\
\hline$[0,0,0]$ GM1-[O3:e:dsp]A_1(a) & 0.00711 & 0.00356 \\
\hline$[0,0,0]$ GM1-[O3:e:dsp]A_2(a) & -0.81762 & 0.40881 \\
\hline$[0,0,0]$ GM1-[O3:e:dsp]A_3(a) & -0.00496 & 0.00248 \\
\hline$[0,0,0] \mathrm{GM} 1-\quad$ all & 2.04668 & \\
\hline
\end{tabular}




\section{References}

(1) Capillas, C.; Tasci, E. S.; Flor, G. D.; Orobengoa, D.; Perez-Mato J. M.; Aroyo, M. I. Z. Krist. 2011, 226, 186.

(2) Bilbao Crystallographic Server: http://www.cryst.ehu.es 